\title{
Effect of 2 Psychotherapies on Depression and Disease Activity in Pediatric Crohn's Disease
}

\section{Citation}

Szigethy, E., A. O. Youk, J. Gonzalez-Heydrich, S. I. Bujoreanu, J. Weisz, D. Fairclough, P. Ducharme, et al. 2015. "Effect of 2 Psychotherapies on Depression and Disease Activity in Pediatric Crohn's Disease." Inflammatory Bowel Diseases 21 (6): 1321-1328. doi:10.1097/ MIB.0000000000000358. http://dx.doi.org/10.1097/MIB.0000000000000358.

\section{Published Version}

doi:10.1097/MIB.0000000000000358

\section{Permanent link}

http://nrs.harvard.edu/urn-3:HUL.InstRepos:17295508

\section{Terms of Use}

This article was downloaded from Harvard University's DASH repository, and is made available under the terms and conditions applicable to Other Posted Material, as set forth at http:// nrs.harvard.edu/urn-3:HUL.InstRepos:dash.current.terms-of-use\#LAA

\section{Share Your Story}

The Harvard community has made this article openly available.

Please share how this access benefits you. Submit a story.

\section{Accessibility}




\title{
Effect of 2 Psychotherapies on Depression and Disease Activity in Pediatric Crohn's Disease
}

\author{
Eva Szigethy, $\mathrm{MD}, \mathrm{PhD},{ }^{*}$ Ada O. Youk, $\mathrm{PhD}^{+}$Joseph Gonzalez-Heydrich, $\mathrm{MD}^{\neq}{ }^{\neq}$Simona I. Bujoreanu, PhD, \\ John Weisz, PhD, Diane Fairclough, DrPH, ${ }^{\S}$ Peter Ducharme, MSW, Neil Jones, PhD, ${ }^{*}$ \\ Francis Lotrich, MD, PhD, ${ }^{*}$ David Keljo, MD, PhD, " Arvind Srinath, MD," Athos Bousvaros, MD, ${ }^{* *}$ \\ David Kupfer, MD,* and David R. DeMaso, $M D^{\neq}$
}

Background: Crohn's disease (CD) is associated with depression. It is unclear if psychosocial interventions offer benefit for depressive symptoms during active $\mathrm{CD}$. In this secondary analysis of a larger study of treating depression in pediatric inflammatory bowel disease, we assessed whether cognitive behavioral therapy (CBT) would differentiate from supportive nondirective therapy in treating depression and disease activity in youth with $\mathrm{CD}$. We also explored whether somatic depressive symptoms showed a different pattern of response in the overall sample and the subset with active inflammatory bowel disease.

Methods: Youth with depression and CD $(\mathrm{n}=161)$ were randomized to 3 months of CBT (teaching coping skills) or supportive nondirective therapy (supportive listening). Depressive severity was measured using the Children's Depression Rating Scale-Revised (CDRS-R) with the somatic depressive subtype consisting of those CDRS-R items, which significantly correlated with CD activity. Disease activity was measured by the Pediatric Crohn's disease Activity Index. Given the potential confound of higher dose steroids, subanalyses excluded subjects on $>20 \mathrm{mg} / \mathrm{d}$ prednisone equivalent ( $\mathrm{n}=34$ ).

Results: Total CDRS-R scores in the overall sample significantly decreased over time after both treatments $(P<0.0001)$. Treatment with CBT was associated with a significantly greater improvement in the Pediatric Crohn's disease Activity Index $(P=0.05)$ and somatic depressive subtype $(P=0.03)$ in those with active inflammatory bowel disease $(\mathrm{n}=95)$ compared with supportive nondirective therapy. After excluding those on steroids $(\mathrm{n}=34)$, there was a significant improvement in total CDRS-R $(P=0.03)$ and in Pediatric Crohn's disease Activity Index $(P=0.03)$ after CBT.

Conclusions: Psychotherapy may be a useful adjunct to treat depression in the context of CD-related inflammation in youth who are not concurrently on higher dose steroids.

(Inflamm Bowel Dis 2015;21:1321-1328)

Key Words: depression, inflammation, physical illness, psychotherapy, inflammatory bowel disease, Crohn's disease, somatic depressive symptoms

G iven that physical illnesses with co-occurring clinical depression are associated with significantly poorer medical outcomes and reduced quality of life in children and adolescents, ${ }^{1-3}$ there is a critical public health need to explore the effectiveness of evidence-based psychiatric interventions on their health care. One such physical illness is inflammatory bowel disease (IBD),

Received for publication December 30, 2014; Accepted January 25, 2015.

From the *Department of Psychiatry, University of Pittsburgh School of Medicine, Pittsburgh, Pennsylvania; 'Department of Biostatistics, University of Pittsburgh, Pittsburgh, Pennsylvania; 'Department of Psychiatry, Boston Children’s Hospital, Boston, Massachusetts; § Department of Psychology, Harvard University, Cambridge, Massachusetts; "Department of Biostatistics and Informatics, Colorado School of Public Health, Aurora, Colorado; 'Department of Pediatric Gastroenterology, Children's Hospital of Pittsburgh UPMC, Pittsburgh, Pennsylvania; and **Department of Pediatric Gastroenterology, Boston Children's Hospital, Boston, Massachusetts.

E. Szigethy currently has a senior investigator research grant from the Crohn's and Colitis Foundation of America (CCFA) and has received funding from NIH Directors Innovator Award (DP2 OD001210), has served as a consultant for Merck and AbbVie Advisor Boards, has received honoraria from Imedex, the CME organizers for the CCFA for educational presentations and is co-editor of the book, Cognitive Behavioral Therapy for Children and Adolescents for which she receives royalties from APPI Press. She is also a consultant for iHOPE Network. J. Weisz is a co-editor for the book, Cognitive Behavioral Therapy for Children and Adolescents. J. Gonzalez-Heydrich has received grant support from the Tommy Fuss Fund, the Al Rashed Family, GlaxoSimthKline, Johnson \& Johnson, Abbott Laboratories, Pfizer Inc., AkzoNobel/Organon, and the NIMH. He has been a consultant for Abbott Laboratories, Pfizer, Johnson \& Johnson (Janssen, McNeil Consumer Health), Novartis, Parke-Davis, GlaxoSmithKline, AstraZeneca, and Seaside Therapeutics. D. Kupfer holds joint ownership of copyright for the Pittsburgh Sleep Quality Index (PSQI); he and his spouse, Dr. Ellen Frank are stockholders in Psychiatric Assessments, Inc. and Health Rhythms, Inc. He is stockholder in AliphCom. Dr. Frank also received royalties from the American Psychological Association and Guilford Press; is a member of the Advisory Board of Servier International, and Editorial Consultant for American Psychiatric Press. The remaining authors have no conflicts of interest to disclose.

Supported by a grant from the National Institute of Mental Health (R01 MH07770) (NCT00534911).

Reprints: Eva Szigethy, MD, PhD, Children's Hospital of Pittsburgh, 4401 Penn Avenue, 3rd Floor Plaza Building, Pittsburgh, PA 15224 (e-mail: szigethye@upmc.edu).

Copyright (C) 2015 Crohn's \& Colitis Foundation of America, Inc.

DOI 10.1097/MIB.0000000000000358

Published online 27 March 2015. 
which is a life-long troubling pediatric physical illness characterized by episodic inflammation of the gastrointestinal tract with bloody diarrhea, abdominal pain, fever, and fatigue. ${ }^{4}$ Treatment of IBD often requires anti-inflammatory agents such as systemic corticosteroids $s^{5}$ and surgery for severe disease activity. Youth with IBD have higher rates of clinical depression compared with healthy children or to children with other chronic physical illnesses. ${ }^{6-8}$

The effects of systemic inflammation on the brain have been conceptualized as one explanation for the relatively higher rates of depression in IBD. ${ }^{9}$ A mechanistic relationship between disease activity and depression has growing support, particularly for clusters of somatic depressive symptoms (e.g., fatigue, sleep disturbance, anhedonia), both in the absence ${ }^{10,11}$ and presence ${ }^{12,13}$ of physical diseases, including IBD. ${ }^{7,14-16}$ In fact, these somatic symptoms may be a marker of more severe disease activity. During periods of extreme disease activity, there may be phylogenetic reasons for organisms to "shut down" to conserve energy, to shift motivated behavior toward recuperation, and care seeking to promote the organism's optimal healing. ${ }^{17}$

Though Crohn's disease (CD) and ulcerative colitis (UC) are characterized as IBD subtypes and share many clinical features, each has different underlying inflammatory pathophysiologies. ${ }^{18,19}$ For example, lesions in CD can involve all layers of the gastrointestinal mucosa and are associated with more systemic inflammation, whereas UC lesions are more superficial with disease activity less frequently reflected by elevated inflammatory markers. Such phenotypic heterogeneity makes it more challenging to evaluate whether cognitive behavioral therapy (CBT) has any selective benefits over supportive nondirective therapy (SNDT). In a recent randomized trial comparing CBT to SNDT for clinically depressed youth with either CD or UC, we found significant improvements for both interventions in depression severity, global functioning, quality of life, and disease activity. ${ }^{20}$ However, the only statistically significant difference between treatments was a greater reduction in the pooled disease activity in the CBT group, in a setting of mild disease activity at baseline.

In states of milder inflammation, depressive symptoms, even somatic ones, have been shown to improve after CBT in youth with IBD in an open trial. ${ }^{21,22}$ These findings are consistent with the concept that teaching coping skills by targeting behavioral activation and cognitive reframing, the 2 predominant components of $\mathrm{CBT}$, may be a viable pathway in improving underlying depression under these conditions.

The current study is a secondary analysis of a subsample of adolescents with $\mathrm{CD}$, representing $74 \%$ of the original sample ${ }^{20}$ with more systemic inflammation due to transmural gut wall involvement. The current study extends our previous work by evaluating whether there were differences in improvement in overall depression, somatic depressive symptoms associated with CD activity, and systemic inflammation between CBT and SNDT interventions. We postulated that both interventions would reduce depression but that CBT would have a greater impact on reducing depression overall in the sample, conceptualizing that taught coping skills, such as behavioral activation and cognitive reframing, would help to make participants more active agents in controlling modifiable factors in their physical illness experience. We hypothesized further that somatic depressive symptoms would show the greatest degree of improvement after CBT in the presence of disease activity. That is, when learning to cope, a participant would be able to counter the brain manifestations of CD more than through nondirective sharing about their illness (SNDT). Because systemic steroids, particularly at higher doses, can also have a confounding effect on treatment outcomes as they have been shown to have an independent negative effect on emotional and cognitive functioning in daily life in treating youth with $\mathrm{IBD},{ }^{23}$ we analyzed the data also excluding those on the higher dose steroids at baseline. We postulated that CBT would be associated with an even greater change in depressive severity in this sample.

\section{MATERIALS AND METHODS}

\section{Participants}

Youth ages 9 to 17 years with either CD or UC and their parents were recruited from Children's Hospital of Pittsburgh and Boston Children's Hospital between 2008 and 2012 using a protocol approved by the Institutional Review Boards at both sites. The eligibility criteria, trial methods, and outcomes have been previously described. ${ }^{20}$ Participants were screened for depression with the Children's Depression Inventory $(\mathrm{CDI})^{24}$; those with CDI scores $\geq 10$ were interviewed within 2 weeks using the Kiddie-Schedule for Affective Disorders and Schizophrenia for Children, Present Version (KSADS-PL). ${ }^{25}$ Youth were included in the trial if they met criteria for major or minor depression by DSM-IV-TR criteria. ${ }^{26}$

\section{Design}

This study represents a secondary analysis of the data collected as part of the randomized trial comparing CBT and SNDT for the treatment of depression and quality of life, functioning, and disease activity in a sample of youth with IBD. In the parent study, eligible subjects with $\mathrm{CD}(\mathrm{n}=161)$ or $\mathrm{UC}(\mathrm{n}=$ 56) were randomized to receive a 12 -week course of either modified CBT or SNDT and were assessed with an identical battery of measures at baseline and at intervention completion ( $3 \mathrm{mo}$ ). This study focuses on the CD cohort using depression and disease activity as outcomes for hypothesis-generating analyses.

\section{Measures}

\section{Depression}

The CDI (child and parent report version) is a 27-item self-report questionnaire assessing the presence and severity of depressive symptoms. ${ }^{24} \mathrm{CDI}$ scores $\geq 10$ on either child or parent rating were considered as reflective of clinically significant depressive symptoms.

The KSADS-PL is a semistructured diagnostic interview for youth and parents assessing for the presence of psychiatric 
disorders based on DSM-IV criteria. ${ }^{25}$ Depression remission was defined as no longer meeting KSADS-PL criteria for depressive diagnosis posttreatment.

The Children's Depression Rating Scale-Revised (CDRS-R), a semistructured clinician-rated instrument assessing depressive severity, ${ }^{27}$ was chosen as the primary emotional outcome because it has been shown to differentiate depressive from physical symptoms. ${ }^{28}$ CDRS-R scores can range between 17 and 113 with values $\leq 28$ are consistent with remitted depression.

\section{Disease Activity}

$\mathrm{CD}$ onset, presentation and course, gastrointestinal tract disease location, medication use (e.g., corticosteroids, biological therapies, and/or immunomodulators), surgical history, and ostomy status were obtained from parents and medical record verification. Subjects with $\geq 20 \mathrm{mg}$ of prednisone or systemic steroid equivalent at baseline were defined as having higher dose steroid utilization. Biomarkers of systemic inflammation erythrocyte sedimentation rate (ESR) and C-reactive protein (CRP) were recorded from the medical record by a blinded gastroenterologist. Pertinent demographic information were recorded from parent reports and verified in the medical record.

The Pediatric Crohn's Disease Activity Index (PCDAI) is a well-validated scale used to determine disease activity in $\mathrm{CD}$ and has been shown to correlate significantly with gastrointestinal inflammation. ${ }^{29,30}$ It measures 3 domains during medical visits: (1) self-report of pain and stool consistency, (2) functional disability, and (3) objective physical/biochemical data (such as ESR, hematocrit, albumin, growth, and physical examination). Rated by a blinded gastroenterologist PCDAI scores $\geq 30$ were considered to indicate moderate disease activity, scores in the range of 11 to 29 reflected mild disease activity, and scores $\leq 10$ were indicative of inactive disease. ${ }^{31}$ Disease activity (PCDAI), ESR, and CRP were extracted from the medical record of a gastroenterology visit only if this visit was within 1 week of the baseline assessment and within 2 weeks of the posttreatment ( $3 \mathrm{mo}$ ) assessment.

\section{Interventions}

Randomized subjects underwent up to 12 weekly sessions of either $\mathrm{CBT}^{22,23}$ or SNDT. Therapists $(\mathrm{n}=10)$ experienced in treating physically ill youth were trained in both manual-based interventions and received weekly supervision. All sessions were audiotaped to allow for quality assurance and to assure treatment fidelity. Each treatment was tailored to the developmental level of each youth. Up to $75 \%$ of sessions could be completed by telephone. More detailed descriptions of each intervention have been described previously. ${ }^{33}$

\section{Statistical Analyses}

Descriptive statistics were computed for all demographic and clinical measures. T-tests were used for continuous variables and chi-square tests (or Fisher's exact tests) for categorical variables to assess baseline differences between the 2 treatment groups. To assess how somatic depressive symptoms responded to psychotherapy, we created a somatic subscale of the CDRS-S by combining the somatic items of the CDRS-R that were statistically significantly correlated with baseline CD activity. The criteria for inclusion were defined as the CDRS-R items, which correlated with total PCDAI score with a coefficient of $>0.2(P<0.01)$.

Linear mixed effects models were used to assess the impact of treatment (CBT versus SNDT) on CDRS-R over time (baseline to $3 \mathrm{mo}$ ) in intent-to-treat analyses. Change from baseline CDRS-R scores was modeled as a function of time and the interaction of treatment and time. Because subjects were randomized into each treatment group (there were no baseline group differences), baseline outcome scores for each treatment were set to be equal in the models by not including the main effect of treatment. Effect sizes for the treatment impact on outcomes of interest were computed using Cohen's d estimates. Similar models were also fit to assess the impact of treatment on disease activity (using PCDAI scores as the outcome) and on the somatic depressive subscale. Exploratory linear mixed models were fit to evaluate separately the change in the depressive outcomes (total CDRS-R and somatic CDRS-R subscale) over time within 2 groups of disease activity: inactive (PCDAI $\leq 15)$ versus active $($ PCDAI $>15)$. Models were also fit within the subgroup of youth who were not on higher dose systemic steroids at baseline.

All models were adjusted for site. Statistical significance was determined using Wald tests (z-tests) from the linear mixed models using $\alpha=0.05$ (two-sided). No correction was made to account for multiple comparisons because all analyses in this study were for hypothesis generation purposes. All analyses were performed using Stata version 12. ${ }^{34}$

The sample size determination for the main study was based on two-tailed tests of hypotheses with size $\alpha=0.05$ (Cohen, 1988; Kraemer, 1997) using a repeated measures design with estimated correlation between the time points of 0.6. The expected effect for CBT for our primary outcome (CDRS-R) was estimated to be large (Cohen's $d>0.8$ ). The expected effect for SNDT for our primary outcome was estimated to have small-tomoderate effect size (range of Cohen's $d=0.2-0.4$ ). The effect size for CBT versus SNDT was then estimated as the difference between these separate effects, thus giving moderate effect sizes of Cohen's $d=0.4$ to 0.6. Based on our current sample size for $\mathrm{CD}$ of $\mathrm{n}=161$, using the original estimated effect sizes, the power ranged from $81 \%$ to $99 \%$.

\section{RESULTS}

\section{Sample Characteristics}

In the parent study, a total of 765 unique subjects with IBD were screened ( 550 with $\mathrm{CD}$ ) with a total of 217 meeting criteria for randomization. From the 161 with $\mathrm{CD}, 82$ were randomized to CBT and 79 to SNDT. At 3 months, 69 youth completed CBT, and 66 completed SNDT treatments. PCDAI information was unavailable for 5 participants at baseline and for 26 participants at month 3 . 
Participant characteristics at baseline are shown in Table 1. Mean age was 14.3 years $(\mathrm{SD}=2.4)$, race was predominantly white $(87.6 \%)$, and there was moderate depressive severity $(\mathrm{CDRS}-\mathrm{R}=46, \mathrm{SD}=12.1)$. Active systemic inflammation was evident by the mildly elevated ESR and CRP. The mean sample PCDAI score of 21.7 (SD = 16.5) was consistent with mild disease activity. In the subgroups consisting of no $(n=119)$ or lowdose (range, 5-15 mg; $\mathrm{n}=8$ ) systemic corticosteroids, the mean PCDAI score was 18.9 (SD = 14.5), consistent with mild disease activity. Analyses were completed on total sample and excluding subjects on $\geq 20 \mathrm{mg} / \mathrm{d}$ prednisone equivalent ( $\mathrm{n}=34$ ). The somatic depressive symptom subtype consisted of CDRS-R items 2 (anhedonia), 4 (sleep), 5 (appetite), 6 (fatigue), 7 (physical complaints), 11 (depressed feelings), and 14 (excessive weeping) (Table 2). Although suicidal ideation (item 13), also correlated with total PCDAI ( $\mathrm{r}=0.17 ; P=0.032$ ), it did not meet the threshold level to be included in the somatic subtype.

Per KSADS-PL, comorbid psychiatric diagnoses included generalized anxiety disorder (20.5\%), specific phobias (14.9\%), attention-deficit hyperactivity disorder $(11.6 \%)$, separation anxiety disorder $(9.7 \%)$, oppositional defiant disorder $(8.9 \%)$, social anxiety disorder $(8.8 \%)$, obsessive compulsive disorder $(1.9 \%)$, dysthymia $(1.4 \%)$, posttraumatic stress disorder $(1.4 \%)$, anorexia nervosa $(0.9 \%)$, panic disorder $(0.5 \%)$, and conduct disorder (0.5\%) (data not shown).

Approximately $75 \%$ of all psychotherapy sessions were delivered by phone instead of face-to-face (data not shown). The most common reason reported by subjects for choosing phone sessions in both therapy arms was the convenience of not having to travel to the hospital. To evaluate whether this choice was due to more severe disease activity, we evaluated the percentage of phone sessions by PCDAI level (inactive versus active) using a linear regression model at baseline with percent phone sessions as the outcome and PCDAI activity level as the independent categorical variable. $C D$ activity was not significantly associated with the percentage of phone sessions $(P=0.052)$ (data not shown).

\section{Intervention Effects on Depressive Severity and Disease Activity}

There were statistically significant differences by site for disease activity $(\mathrm{PCDAI})$ at baseline $(P<0.0001)$ and at month 3 $(P=0.002)$ with the Pittsburgh site having more severe disease.

TABLE 1. Baseline Demographic, Clinical, and Psychosocial Characteristics of Patients with CD by Treatment Group $(\mathrm{n}=161)$

\begin{tabular}{|c|c|c|c|c|c|c|c|}
\hline Measure & \multicolumn{2}{|c|}{ Total Sample $(\mathrm{n}=161)$} & \multicolumn{2}{|c|}{$\mathrm{CBT}(\mathrm{n}=82)$} & \multicolumn{2}{|c|}{ SNDT $(n=79)$} & Statistic, $P$ \\
\hline Age & 161 & $14.3(2.4)$ & 82 & $14.1(2.6)$ & 79 & $14.4(2.3)$ & $\mathrm{t}=-0.85,0.39$ \\
\hline Gender (male) & $75(46.6)$ & - & $41(50.0)$ & - & $34(43.0)$ & - & $\chi^{2}(1)=0.78,0.38$ \\
\hline Site (Pittsburgh) & $104(64.6)$ & - & $53(64.6)$ & - & $51(64.6)$ & - & $\chi^{2}(1)=0.001,0.99$ \\
\hline \multicolumn{8}{|l|}{ Depression } \\
\hline CDI Child (Self-Report Depression) & 160 & $13.59(6.72)$ & 81 & $13.05(6.64)$ & 79 & $14.15(6.80)$ & $\mathrm{t}=-1.04,0.30$ \\
\hline CDI Parent (Self-Report Depression) & 160 & $14.61(6.45)$ & 82 & $14.61(6.26)$ & 78 & $14.60(6.69)$ & $\mathrm{t}=0.01,0.99$ \\
\hline CDRS-R & 161 & $46.01(12.05)$ & 82 & $44.31(11.64)$ & 79 & $47.76(12.28)$ & $\mathrm{t}=-1.82,0.07$ \\
\hline Major Depression & $103(64.0)$ & - & $52(63.4)$ & - & $51(64.6)$ & - & $\chi^{2}(1)=0.02,0.88$ \\
\hline Minor Depression & $58(36.0)$ & & $30(36.6)$ & & $28(35.4)$ & & \\
\hline CRP & 135 & $1.54(2.68)$ & 68 & $1.55(3.08)$ & 67 & $1.53(2.23)$ & $\mathrm{t}=0.04,0.97$ \\
\hline \multicolumn{8}{|l|}{ CD medications } \\
\hline Systemic corticosteroids & $34(21.1)$ & - & $17(20.7)$ & - & $17(21.5)$ & - & $\chi^{2}(1)=0.02,0.90$ \\
\hline Biological therapies & $47(29.2)$ & - & $22(26.8)$ & - & $25(31.7)$ & - & $\chi^{2}(1)=0.45,0.50$ \\
\hline Immunomodulators & $84(52.2)$ & - & $42(51.2)$ & - & $42(53.2)$ & - & $\chi^{2}(1)=0.06,0.81$ \\
\hline Surgery (yes) & $17(10.7)$ & - & $4(4.9)$ & - & $13(16.7)$ & - & FE, $P=0.02$ \\
\hline Ostomy (yes) & $4(2.5)$ & - & $0(0.0)$ & - & $4(5.1)$ & - & $\mathrm{FE}, P=0.06$ \\
\hline
\end{tabular}

FE, Fisher's exact. 
TABLE 2. Correlation Between Individual Depressive Symptoms on CDRS and PCDAl at Baseline

\begin{tabular}{lcrc}
\hline CDRS-R Item & Description & $\mathrm{r}$ & $P$ \\
\hline 2 & Anhedonia & $\mathbf{0 . 2 2}$ & $\mathbf{0 . 0 0 7}$ \\
4 & Appetite & $\mathbf{0 . 2 3}$ & $\mathbf{0 . 0 0 5}$ \\
5 & Sleep & $\mathbf{0 . 3 4}$ & $\mathbf{0 . 0 0 1}$ \\
6 & Fatigue & $\mathbf{0 . 2 3}$ & $\mathbf{0 . 0 0 5}$ \\
7 & Physical complaints & $\mathbf{0 . 3 5}$ & $\mathbf{0 . 0 0 1}$ \\
11 & Depressed mood & $\mathbf{0 . 2 6}$ & $\mathbf{0 . 0 0 2}$ \\
14 & Weeping & $\mathbf{0 . 3 3}$ & $\mathbf{0 . 0 0 1}$ \\
1 & Schoolwork & 0.05 & 0.546 \\
3 & Social withdrawal & 0.01 & 0.915 \\
8 & Irritability & 0.03 & 0.701 \\
9 & Guilt & -0.04 & 0.603 \\
10 & Self esteem & -0.06 & 0.475 \\
12 & Morbid ideation & 0.03 & 0.680 \\
13 & Suicidality & 0.17 & 0.032
\end{tabular}

Pearson's correlation coefficients (r) and respective $P$ values are presented with bolded values representing the somatic/mood symptoms showing significant association at $P<$ 0.01 level.

There were no statistically significant differences between sites for steroid use (data not shown).

For the total CD sample, both treatments showed similar improvement in depressive severity (CDRS-R), with no significant difference between the interventions $(\mathrm{z}=1.78, P=0.07$; Table 3). CDRS-R significantly improved over time for both CBT $(\beta=-15.26 ; \mathrm{z}=-9.28 ; P<0.0001)$ and $\operatorname{SNDT}(\beta=-14.46$; $\mathrm{z}=-7.71 ; P<0.0001$ ) (data not shown).

Within categories of baseline disease severity (inactive versus active), no statistically significant differences in CDRS-R between the treatments were found for the whole sample $(P=0.07$ for active disease, favoring CBT and $P=0.40$ for inactive disease). Both CBT and SNDT resulted in reductions in CDRS-R for both disease activity groups.

To evaluate whether there were differences to psychotherapy response to symptoms more related to disease activity (somatic depressive symptoms) over time, factor-specific linear mixed models were fit using the somatic depressive symptom subtype as the outcome in the total sample and within each PCDAI activity (inactive versus active). There was no specific treatment effect for the somatic depressive symptoms in the total sample or those with inactive $\mathrm{CD}$; however, somatic symptoms showed a significantly greater improvement with CBT in the subgroup with active $\mathrm{CD}(P=0.03)$.

For the total CD sample, there was a significant change in PCDAI over time for CBT $(\beta=-11.64 ; \mathrm{z}=-5.35 ; P<0.0001)$ and for $\operatorname{SNDT}(\beta=-7.31 ; \mathrm{z}=-3.12 ; P=0.002)$ (data not shown). CBT had a selective significant impact on improving PCDAI ( $\mathrm{z}=1.97, P=0.05$; Table 3$)$ as well as ESR $(P=0.004$; $\mathrm{n}=103$; data not shown). CBT was also favored but not statistically significant for decreasing $\mathrm{CRP}(P=0.07 ; \mathrm{n}=97$; data not shown). The total baseline PCDAI score was significantly correlated with both CRP $(\mathrm{r}=0.32, P=0.0002 ; \mathrm{n}=135)$ and ESR (r = 0.50; $P<0.00001 ; \mathrm{n}=148)$.

When youth on systemic steroids $(\geq 20 \mathrm{mg} / \mathrm{d}$ ) at baseline $(n=34)$ were excluded, both interventions again significantly improved depressive severity and disease activity (Table 3 ) for the whole subsample. However, CBT had a significant greater impact than SNDT on reducing total CDRS-R $(\mathrm{z}=2.18, P=$ $0.03)$ and somatic depressive symptoms $(\mathrm{z}=2.14, P=0.03)$ and on improving PCDAI $(\mathrm{z}=2.15, P=0.03)$.

\section{DISCUSSION}

Our findings show that in youth with $\mathrm{CD}$, both CBT and SNDT were associated with a reduction of depression and disease activity over 3 months of psychiatric treatment with only $\mathrm{CD}$ activity and systemic inflammation (ESR) demonstrating a statistically significant improvement after CBT compared with SNDT. These results are similar to what we found in the original study sample, which included youth with $\mathrm{UC},{ }^{20}$ and suggest that the modality of psychotherapy used for depression in chronic pediatric physical illness is not important. Even when youth with CD were stratified into more homogeneous subgroups (those with and without active $\mathrm{CD}$ at baseline), there was no statistically significant difference in the change in depressive severity between the 2 psychotherapies. Given the association that has been found between depressive severity and IBD activity, ${ }^{7,35}$ it was surprising that depressive severity did not parallel changes in $\mathrm{CD}$ activity by not showing a statistically significant greater improvement after CBT.

Depression is a heterogeneous entity with different depressive symptom clusters associated with different clinical factors. Our previous work using person-centered analyses to characterize different depressive subtypes in a predominantly CD cohort found that youth with a depressive subtype consisting of somatic depressive symptoms had higher levels of inflammation than those with other types of depressive profiles. ${ }^{36}$ Somatic depressive symptoms have been reported to be associated with inflammation both in animal models of colitis and humans with other conditions linked to inflammatory processes such as patients with hepatitis treated with proinflammatory protein (interferon, an exogenous cytokine). ${ }^{37,38}$

We evaluated the somatic depressive symptoms which significantly correlated with $\mathrm{CD}$ activity to assess whether there were differential psychotherapy effects favoring CBT. Our analyses showed that mood symptoms and somatic symptoms (e.g., anhedonia, fatigue, sleep disturbance, and appetite changes) were most significantly correlated with active $\mathrm{CD}$ and likely represent the effects of immune activation in the brain. In examining changes in these somatic symptoms to psychotherapy, although there was no statistically significant difference between treatments in the total CD sample, there was a statistically 
TABLE 3. Effect of Treatment (CBT Versus SNDT) Over Time Controlling for Site

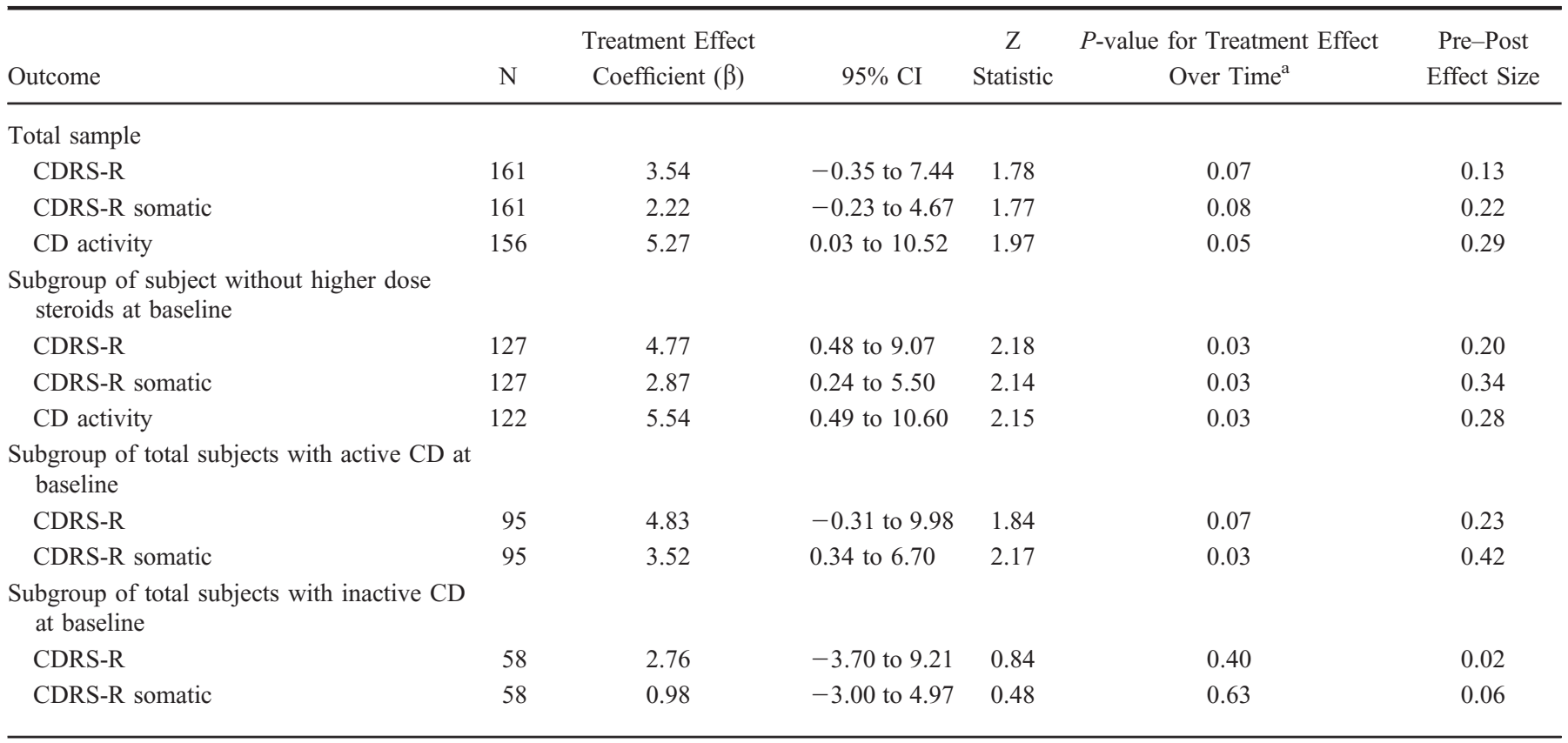

${ }^{\mathrm{a} C B T}$ was favored over SNDT in all of these outcomes.

significant difference favoring CBT in the 95 youth with active $\mathrm{CD}$ (Fig. 1). These findings suggest that in the context of active IBD, depressed adolescents with a preponderance of somatic depressive symptoms have a greater benefit from CBT, whereas those in remission or with other types of depressive symptoms benefit more generally from psychosocial interventions that are not modality specific. A review of brain-gut interactions in IBD suggests that psychosocial interventions may have a role in treating neuropsychiatric symptoms associated with inflammation by altering stress response and by the anti-inflammatory effects of vagal activation. ${ }^{9}$

The differential improvement of depression after CBT versus SNDT in the context of disease activity is clinically relevant. We conceptualize that a more didactic therapeutic approach which targets behavioral activation, active problem solving, relaxation, and cognitive reframing as ways of improving perceived control may be more useful than the more empathic active listening ingredients of the supportive therapy. Although psychotherapy studies of depressed adolescents without physical illness identified some of these skills, e.g., exercise, problem solving, improving sense of self-efficacy as "active ingredients," 39 future component analysis of the psychotherapy content and process will be needed to evaluate which specific CBT skills most benefited which subset of depressed adolescents with IBD.

In the current study, the selective association of somatic symptom severity with baseline inflammatory activity and the statistically significant improvement in inflammation (ESR) after CBT treatment support the notion that the improvement in disease activity was related to inflammation-related IBD symptoms. These results replicate our previous findings of CBT effects on IBD activity in youth with subsyndromal depression ${ }^{22}$ and are also consistent with findings in a depressed adult cohort where depression improved significantly more with anti-inflammatory drug treatment compared with placebo only in the setting of elevated CRP. ${ }^{40}$ Thus, it is conceivable that depressed youth with mild-to-moderate IBD activity are similar to depressed patients who have mild elevation in circulating inflammatory biomarkers but no systemic disease. Future studies are needed to investigate psychotherapy effects under these conditions and effects on specific depressive symptoms such as anhedonia or fatigue.

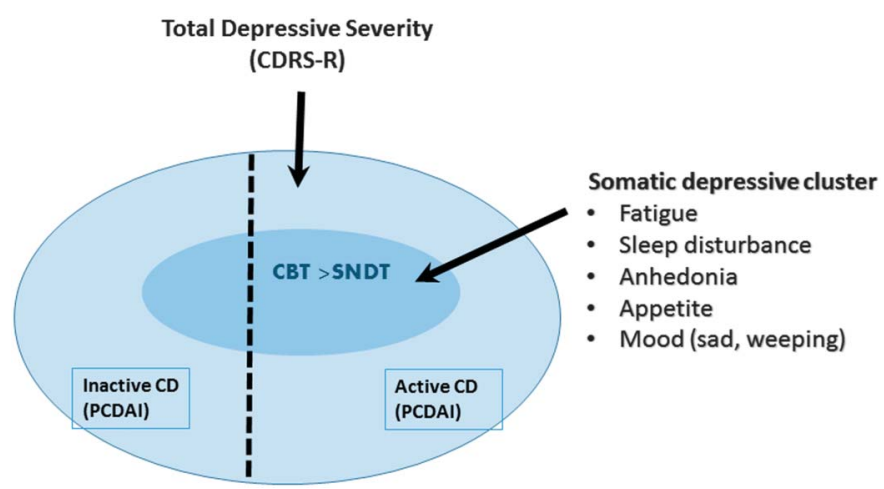

FIGURE 1. CBT associated with more significant improvement in somatic depressive symptoms in the presence of active CD compared with SNDT. 
One proposed pathway of action for the differential effect of CBT in the context of systemic inflammation is by increasing the parasympathetic tone. Vagal stimulation, in patients with rheumatoid arthritis and in animal models of colitis, has been shown to have potent anti-inflammatory effects, ${ }^{9,41-44}$ and there is a growing literature supporting anti-inflammatory effects of other types of psychosocial interventions, ${ }^{45-47}$ although the underlying pathways remain to be elucidated. Future studies evaluating treatment mediators will need to target such mechanisms ${ }^{9,45}$ by replicating these findings in larger samples recruited and randomized based on depressive subtypes or profiles.

Systemic steroids at higher doses can have confounding direct negative effects on the brain. ${ }^{23}$ When the 34 youth on higher dose steroids were systematically excluded from analyses, there was a significantly greater improvement in overall depressive severity in the total sample and in those with active IBD after CBT. Systemic steroids are often used to treat severe IBD flares, and thus this steroid subgroup could represent the subgroup where depression is serving a protective effect to facilitate healing and thus is not as amendable to psychosocial interventions.

Although no randomized trials of antidepressant treatment have been conducted with patients with IBD, animal models of colitis using several different classes of antidepressants (e.g., serotonin reuptake inhibitors, bupropion) have shown that these antidepressants have anti-inflammatory properties in the gastrointestinal tract. ${ }^{48-50}$ Studies of serotonergic antidepressants in other models of inflammatory depression, however, have not shown positive effects on somatic symptoms. ${ }^{16}$ In adults with $\mathrm{CD}$, an open trial of bupropion targeting dopaminergic circuits showed favorable effects on both depression and CD activity. ${ }^{51,52}$ Interestingly, dopaminergic pathways connecting basal ganglia with cortical regions have been implicated in other models of inflammatory depression ${ }^{33,53,54}$ and may be one possible pathway by which CBT exerts an effect. Again, future studies will need to evaluate the role of psychotropic agents as an adjunct to psychotherapy in decreasing inflammation and depression in this population (e.g., fatigue, sleep disturbance, pain).

A potential limitation of the study is that $C D$ activity was recorded from the medical charts and not as part of the research protocol, so there are subjects with missing data. However, the $\mathrm{CD}$ activity data which was available from the clinical record at the time of the depressive screen was reviewed by a gastroenterologist blinded to study therapy assignment. Another possible limitation is the lack of adjustment for the overall level of statistical significance of the study for multiple comparisons as the study was hypothesis-generating in nature. ${ }^{55}$ However, the importance of our findings goes beyond statistical significance given its clinical relevance for the development of concept-driven interventions focused on understanding the treatment of depression in inflammatory conditions. The finding of increased CBT efficacy under conditions of mild inflammation can have applicability for patients with endogenous depression who may have mildly elevated systemic inflammation. ${ }^{40}$ Although it is possible that improvement of depression over time in both psychotherapeutic treatments represents regression to the mean independent of the effects of either intervention, the favoring of CBT in these subgroups of patients argues against an absence of impact. Adding placebo-controlled or treatment as usual arms in randomized treatment trials would help to resolve this question. Finally, larger samples would also allow for more rigorous testing of treatment response confounders such as steroids, biologics, and other immunomodulatory agents used to treat $\mathrm{CD}$.

\section{CONCLUSIONS}

Depression in physical illnesses, like IBD, is a significant public health problem that is common, disrupts normal development, and causes substantial functional impairment. This study provides evidence that psychotherapy, regardless of the specific modality, can be a useful adjunct the health care of youth with IBD both in addressing associated depression and illness severity. More specifically, CBT may be best fitted to treat overall depression and somatic depressive symptoms in youth with inflammation, whereas empathic support and/or CBT can help during periods of inactive or more severe inflammation.

\section{ACKNOWLEDGMENTS}

The authors thank Margaret Kirshner, Melissa Newara, F. Nicole McCarthy, and Jennifer Holland for their support as research specialists. Both psychotherapy treatment manuals are available from Dr. Szigethy.

\section{REFERENCES}

1. Bennett DS. Depression among children with chronic medical problems: a meta-analysis. J Pediatr Psychol. 1994;19:149-169.

2. Blackman JA, Conaway MR. Developmental, emotional and behavioral co-morbidities across the chronic health condition spectrum. J Pediatr Rehabil Med. 2013;6:63-71.

3. Verhoof E, Maurice-Stam H, Heymans H, et al. Health-related quality of life, anxiety and depression in young adults with disability benefits due to childhood-onset somatic conditions. Child Adolesc Psychiatry Ment Health. 2013;7:12.

4. Regueiro MD, Swoger JM. Clinical Challenges and Complications of IBD. Thorofare, NJ: SLACK Inc; 2013.

5. Michail S, Ramsy M, Soliman E. Advances in inflammatory bowel diseases in children. Minerva Pediatr. 2012;64:257-270.

6. Burke P, Meyer V, Kocoshis S, et al. Depression and anxiety in pediatric inflammatory bowel disease and cystic fibrosis. J Am Acad Child Adolesc Psychiatry. 1989;28:948-951.

7. Szigethy E, Levy-Warren A, Whitton S, et al. Depressive symptoms and inflammatory bowel disease in children and adolescents: a cross-sectional study. J Pediatric Gastroenterology Nutrition. 2004;39:395-403.

8. Greenley RN, Hommel KA, Nebel J, et al. A meta-analytic review of the psychosocial adjustment of youth with inflammatory bowel disease. J Pediatr Psychol. 2010;35:857-869.

9. Bonaz BL, Bernstein CN. Brain-gut interactions in inflammatory bowel disease. Gastroenterology. 2013;144:36-49.

10. Gabbay V, Klein RG, Alonso CM, et al. Immune system dysregulation in adolescent major depressive disorder. J Affect Disord. 2009;115:177-182.

11. Mills NT, Scott JG, Wray NR, et al. Research review: the role of cytokines in depression in adolescents: a systematic review. J Child Psychol Psychiatry. 2013;54:816-835.

12. Hood KK, Lawrence JM, Anderson A, et al. Metabolic and inflammatory links to depression in youth with diabetes. Diabetes Care. 2012;35: $2443-2446$. 
13. Shanahan L, Copeland WE, Worthman CM, et al. Children with both asthma and depression are at risk for heightened inflammation. J Pediatr. 2013.

14. Dantzer R, Kelley KW. Twenty years of research on cytokine-induced sickness behavior. Brain Behav Immun. 2007;21:153-160.

15. Biesmans S, Meert TF, Bouwknecht JA, et al. Systemic immune activation leads to neuroinflammation and sickness behavior in mice. Mediators Inflamm. 2013;2013:271359.

16. Capuron L, Gumnick JF, Musselman DL, et al. Neurobehavioral effects of interferon-alpha in cancer patients: phenomenology and paroxetine responsiveness of symptom dimensions. Neuropsychopharmacology. 2002;26:643-652.

17. Tsuru S, Nomoto K, Taniguchi M, et al. Depression of protective mechanism during the early phase of a viral infection in tumor-bearing mice and prevention by PSK. Cancer Immunol Immunother. 1986;22:114-118.

18. Bousvaros A, Antonioli DA, Colletti RB, et al. Differentiating ulcerative colitis from Crohn's disease in children and young adults: report of a working group of the North American Society for Pediatric Gastroenterology, Hepatology, and Nutrition and the Crohn's and Colitis Foundation of America. J Pediatric Gastroenterology Nutrition. 2007;44:653-674.

19. Ponder A, Long MD. A clinical review of recent findings in the epidemiology of inflammatory bowel disease. Clin Epidemiol. 2013;5:237-247.

20. Szigethy E, Bujoreanu SI, Youk AO, et al. Randomized efficacy trial of two psychotherapies for depression in youth with inflammatory bowel disease. J Am Acad Child Adolesc Psychiatry. 2014;53:726-735.

21. Szigethy E, Whitton SW, Levy-Warren A, et al. Cognitive-behavioral therapy for depression in adolescents with inflammatory bowel disease: a pilot study. J Am Acad Child Adolesc Psychiatry. 2004;43:1469-1477.

22. Szigethy E, Kenney E, Carpenter J, et al. Cognitive-behavioral therapy for adolescents with inflammatory bowel disease and subsyndromal depression. J Am Acad Child Adolesc Psychiatry. 2007;46:1290-1298.

23. Mrakotsky C, Forbes PW, Bernstein JH, et al. Acute cognitive and behavioral effects of systemic corticosteroids in children treated for inflammatory bowel disease. J Int Neuropsychol Soc. 2013;19:96-109.

24. Kovacs M. The Children's Depression, Inventory (CDI). Psychopharmacol Bull. 1985;21:995-998.

25. Kaufman J, Birmaher B, Brent D, et al. Schedule for Affective Disorders and Schizophrenia for School-Age Children-Present and Lifetime Version (K-SADS-PL): initial reliability and validity data. J Am Acad Child Adolesc Psychiatry. 1997;36:980-988.

26. American Psychiatric A, American Psychiatric Association. Task Force on D-I. Diagnostic and Statistical Manual of Mental Disorders: DSM-IV-TR. Washington, DC: American Psychiatric Association; 2000.

27. Poznanski EO, Mokros HB. Children's Depression Rating Scale, Revised (CDRS-R) Manual. Los Angeles, CA: Western Psychological Services; 1996.

28. Morrison KM, Goli A, Van Wagoner J, et al. Depressive symptoms in inner-city children with asthma. Prim Care Companion J Clin Psychiatry. 2002;4:174-177.

29. Harms HK, Blomer R, Bertele-Harms RM, et al. A paediatric Crohn's disease activity index (PCDAI). Is it useful? Study Group on Crohn's Disease in Children and Adolescents. Acta Paediatr Suppl. 1994;83:22-26.

30. Loonen HJ, Griffiths AM, Merkus MP, et al. A critical assessment of items on the Pediatric Crohn's Disease Activity Index. J Pediatric Gastroenterology Nutrition. 2003;36:90-95.

31. Turner D, Griffiths AM, Walters TD, et al. Appraisal of the pediatric Crohn's disease activity index on four prospectively collected datasets: recommended cutoff values and clinimetric properties. Am J Gastroenterol. 2010;105:2085-2092.

32. Szigethy E, Thompson R, Turner S, et al. Cognitive behavioral therapy for general medical conditions. In: E S, Weisz JR, Findling RL, eds. Cognitive Behavioral Therapy for Children and Adolescents. Washington, DC: American Psychiatric Publishing; 2012:331-382.

33. Eisenberger NI, Berkman ET, Inagaki TK, et al. Inflammation-induced anhedonia: endotoxin reduces ventral striatum responses to reward. Biol Psychiatry. 2010;68:748-754.

34. Stata Statistical Software: Release 12 [Computer Program]. College Station, TX: StataCorp LP; 2011.
35. Goodhand JR, Wahed M, Mawdsley JE, et al. Mood disorders in inflammatory bowel disease: relation to diagnosis, disease activity, perceived stress, and other factors. Inflamm Bowel Dis. 2012.

36. Szigethy EM, Youk AO, Benhayon D, et al. Depression subtypes in pediatric inflammatory bowel disease. J Pediatric Gastroenterology Nutrition. 2014;58:574-581.

37. Miller AH, Maletic V, Raison CL. Inflammation and its discontents: the role of cytokines in the pathophysiology of major depression. Biol Psychiatry. 2009;65:732-741.

38. Franzen PL, Buysse DJ, Rabinovitz M, et al. Poor sleep quality predicts onset of either major depression or subsyndromal depression with irritability during interferon-alpha treatment. Psychiatry Res. 2010;177: 240-245.

39. Spirito A, Esposito-Smythers C, Wolff J, et al. Cognitive-behavioral therapy for adolescent depression and suicidality. Child Adolesc Psychiatr Clin N Am. 2011;20:191-204.

40. Raison CL, Rutherford RE, Woolwine BJ, et al. A randomized controlled trial of the tumor necrosis factor antagonist infliximab for treatmentresistant depression: the role of baseline inflammatory biomarkers. JAMA Psychiatry. 2013;70:31-41.

41. Ghia JE, Blennerhassett P, Kumar-Ondiveeran H, et al. The vagus nerve: a tonic inhibitory influence associated with inflammatory bowel disease in a murine model. Gastroenterology. 2006;131:1122-1130.

42. Ghia JE, Blennerhassett P, Collins SM. Impaired parasympathetic function increases susceptibility to inflammatory bowel disease in a mouse model of depression. J Clin Invest. 2008;118:2209-2218.

43. Koopman FA, Stoof SP, Straub RH, et al. Restoring the balance of the autonomic nervous system as an innovative approach to the treatment of rheumatoid arthritis. Mol Med. 2011;17:937-948.

44. Ghia JE, Blennerhassett P, El-Sharkawy RT, et al. The protective effect of the vagus nerve in a murine model of chronic relapsing colitis. Am J Physiol Gastrointest Liver Physiol. 2007;293:G711-G718.

45. Mawdsley J, Jenkins D, Macey M, et al. The effect of hypnosis on systemic and rectal mucosal measures of inflammation in ulcerative colitis. Am J Gastroenterol. 2008;103:1460-1469.

46. Rosenkranz MA, Davidson RJ, Maccoon DG, et al. A comparison of mindfulness-based stress reduction and an active control in modulation of neurogenic inflammation. Brain Behav Immun. 2013;27:174-184.

47. Schoen M, Nowack K. Reconditioning the stress response with hypnosis CD reduces the inflammatory cytokine IL-6 and influences resilience: a pilot study. Complement Ther Clin Pract. 2013;19:83-88.

48. Rahimi HR, Shiri M, Razmi A. Antidepressants can treat inflammatory bowel disease through regulation of the nuclear factor-kappaB/nitric oxide pathway and inhibition of cytokine production: a hypothesis. World $J$ Gastrointest Pharmacol Ther. 2012;3:83-85.

49. Daneshmand A, Mohammadi H, Rahimian R, et al. Chronic lithium administration ameliorates 2,4,6-trinitrobenzene sulfonic acid-induced colitis in rats; potential role for adenosine triphosphate sensitive potassium channels. J Gastroenterol Hepatol. 26:1174-1181.

50. Brustolim D, Ribeiro-dos-Santos R, Kast RE, et al. A new chapter opens in anti-inflammatory treatments: the antidepressant bupropion lowers production of tumor necrosis factor-alpha and interferon-gamma in mice. Int Immunopharmacol. 2006;6:903-907.

51. Kane S, Altschuler EL, Kast RE. Crohn's disease remission on bupropion. Gastroenterology. 2003;125:1290.

52. Kast R. Anti- and pro-inflammatory considerations in antidepressant use during medical illness: bupropion lowers and mirtazapine increases circulating tumor necrosis factor-alpha levels. Gen Hosp Psychiatry. 2003;25: 495-496.

53. Felger JC, Miller AH. Cytokine effects on the basal ganglia and dopamine function: the subcortical source of inflammatory malaise. Front Neuroendocrinol. 2012;33:315-327.

54. Miller AH, Haroon E, Raison CL, et al. Cytokine targets in the brain: impact on neurotransmitters and neurocircuits. Depress Anxiety. 2013;30: 297-306.

55. Feise RJ. Do multiple outcome measures require p-value adjustment? BMC Med Res Methodol. 2002;2:8. 